\title{
Modeling the Performance of an Integrated Photoelectrolysis System with $10 \times$ Solar Concentrators
}

\author{
Yikai Chen, Chengxiang Xiang, Shu Hu, and Nathan S. Lewis*,z \\ Beckman Institute, Kavli Nanoscience Institute, and Joint Center for Artificial Photosynthesis, Division of Chemistry \\ and Chemical Engineering, California Institute of Technology, Pasadena, California 91125, USA
}

\begin{abstract}
Two designs for an integrated photoelectrolysis system that uses a $10 \times$ concentrating solar collector have been investigated in detail. The system performance was evaluated using a multi-physics model that accounted for the properties of the tandem photoabsorbers, mass transport, and the electrocatalytic performance of the oxygen-evolution and hydrogen-evolution reactions (OER and HER, respectively). The solar-to-hydrogen (STH) conversion efficiencies and the ohmic losses associated with proton transport in the solution electrolyte and through the membrane of the photoelectrolysis system were evaluated systematically as a function of the cell dimensions, the operating temperatures, the bandgap combinations of the tandem cell, and the performance of both the photoabsorbers and electrocatalysts. Relative to designs of optimized systems that would operate without a solar concentrator, the optimized $10 \times$ solar concentrator designs possessed larger ohmic losses and exhibited less uniformity in the distribution of the current density along the width of the photoelectrode. To minimize resistive losses while maximizing the solar-to-hydrogen conversion efficiency, $\eta_{\mathrm{STH}}$, both of the designs, a two-dimensional "trough" design and a three-dimensional "bubble wrap" design, required that the electrode width or diameter, respectively, was no larger than a few millimeters. As the size of the electrodes increased beyond this limiting dimension, the $\eta_{\mathrm{STH}}$ became more sensitive to the performance of the photoabsorbers and catalysts. At a fixed electrode dimension, increases in the operating temperature reduced the efficiency of cells with smaller electrodes, due to degradation in the performance of the photoabsorber with increasing temperature. In contrast, cells with larger electrode dimensions showed increases in efficiency as the temperature increased, due to increases in the rates of electrocatalysis and due to enhanced mass transport. The simulations indicted that cells that contained 10\% photoabsorber area, and minimal amounts of Nafion or other permselective membranes (i.e. areal coverages and volumetric fractions of only a few percent of the cell), with the remaining area comprised of a suitable, low-cost inert, non porous material (flexible polymers, inert inorganic materials, etc.) should be able to produce high values of $\eta_{\text {STH }}$, with $\eta_{\mathrm{STH}}=29.8 \%$ for an optimized design with a bandgap combination of $1.6 \mathrm{eV} / 0.9 \mathrm{eV}$ in a tandem photoabsorber system at $350 \mathrm{~K}$. (C) The Author(s) 2014. Published by ECS. This is an open access article distributed under the terms of the Creative Commons Attribution 4.0 License (CC BY, http://creativecommons.org/licenses/by/4.0/), which permits unrestricted reuse of the work in any medium, provided the original work is properly cited. [DOI: 10.1149/2.0751410jes] All rights reserved.
\end{abstract}

Manuscript submitted June 13, 2014; revised manuscript received July 10, 2014. Published August 1, 2014.

Artificial photosynthesis could provide a promising route to largescale solar energy conversion and storage. ${ }^{1-4}$ Recent techno-economic studies have evaluated various designs for integrated photoelectrolysis systems, including a very promising system that makes use of concentrated illumination. ${ }^{5,6}$ A discrete III-V photovoltaic cell connected electrically in series with a discrete polymer-electrolyte membrane (PEM) electrolyzer has demonstrated a solar-to-hydrogen (STH) conversion efficiency of $18 \%$ under 500 Suns. $^{7}$ Although concentrated photovoltaics (CPV) typically incorporate multi-stage optical systems to achieve high optical concentration ( $~ 400$ Suns to 1200 Suns $),{ }^{8-10}$ integrated photoelectrochemical systems for large scale, distributed solar-to-fuel applications are most likely operate efficiently and scalably at lower solar concentration factors $(5-100)$ due to limitations associated with electrocatalytic overpotential losses, ohmic losses, and mass transport restrictions associated with high current densities in a system operating under very concentrated sunlight. Notably, systems that utilize a low-multiple concentrating solar collector, such as a $10 \times$ concentrator, requires little or no active solar tracking or temperatureregulation systems. ${ }^{11-13}$ Conceptual designs of coupling low concentrator solar collectors with a photoelectrochemical cell have been proposed. ${ }^{14,15}$ A principal advantage of a sunlight-concentrating design for a solar-to-fuels generator is the potential reduction of the usage per unit area of photoabsorber materials, which could result in a significant reduction in the system cost. ${ }^{5,6}$ Although extensive modeling and simulation efforts have been completed for solar fuel generator system designs without solar concentrators, including various operating conditions and using different types of input feed-stocks including liquid electrolytes ${ }^{16-18}$ and water vapor feeds ${ }^{19}$, the design criteria and constraints for an integrated system that exploits concentrating solar collectors have not yet been evaluated.

The high-intensity illumination, and the expected elevated operating temperatures in a light-concentrating photoelectrolysis system, could have significant impacts on the performance of the individual

*Electrochemical Society Active Member.

${ }^{\mathrm{z} E}$-mail: nslewis@caltech.edu components of the system, and thus on the efficiency of the system as a whole. Increases in the illumination intensity would increase the photocurrent density and would concomitantly improve the opencircuit voltage and the fill factor of the current-voltage characteristic of the photoabsorber materials. ${ }^{20}$ The increased current density would also, however, result in an increase in the ohmic losses of the cell, as well as produce an increase to the overpotentials required to drive the oxygen-evolution reaction (OER) and the hydrogen-evolution reaction (HER). ${ }^{21}$ Increases in the operating temperatures would introduce similar trade-offs to the system design, because the increased temperature would degrade the performance of the semiconducting light absorbers while improving both the mass transport in the electrolyte and the performance of the HER and OER catalysts. As a result, the overall system efficiency as a function of the cell geometry, the illumination intensity, and the operating temperature depends upon the working principles for each component as well as upon the detailed mathematical relationships between the components.

Previously reported results from the modeling of integrated photoelectrolysis system designs without solar concentrators have shown that the geometric parameters of the cell dominate the cell performance. Specifically, the width of the photoelectrode must be less than a few centimeters to minimize the ohmic losses from the ionic transport in the electrolyte and membrane. ${ }^{17,22}$ At the higher operating current density produced by concentrated sunlight, the details of the cell geometry are likely to play an even more important role. Although a smaller cell will typically reduce the efficiency losses due to mass transport in the electrolyte, the assembly and integration of absorber materials and other system components could be easier with macroscopic, rather than microscopic, components. Thus, an optimal design would likely maximize the photoelectrode width while minimizing any efficiency losses due to mass transport limitations and ohmic losses.

In this work, two types of integrated photoelectrolysis systems that use a concentrated light source have been investigated: a twodimensional "trough" design and a three-dimensional "bubble wrap" design. The concentration of the illumination was chosen to be ten-fold relative to natural sunlight. The solar-to-hydrogen $(\mathrm{STH})$ conversion 
efficiency for the systems, and the ohmic loss as a function of the cell geometry, operating temperatures, and catalyst performance were evaluated systematically using a fully coupled model for the system as a whole. We have also evaluated the requirements for the size and placement of a proton-exchange membrane, such as Nafion, to minimize the usage of this material, as well as of analogous highperformance polymers, as the permselective gas-blocking component of the system.

\section{Modeling}

Cell designs. - Figure 1 presents schematically the two cell designs that were modeled in this work. Both designs include a $10 \times$ solar concentrator and an integrated photoelectrolysis assembly. The detailed configuration and construction of the solar concentrator is beyond the scope of this work, but two-dimensional or three-dimensional Fresnel lens, compound parabolic concentrators (CPC), or dielectric totally internally reflecting concentrators could be employed to achieve the $10 \times$ solar concentration specified in the designs. For example, with ten-fold concentration, efficient light collection up to 7.5 hours/day can be achieved by compound parabolic concentrators only with occasional tilt adjustments. ${ }^{23}$ The two-dimensional "trough" de-
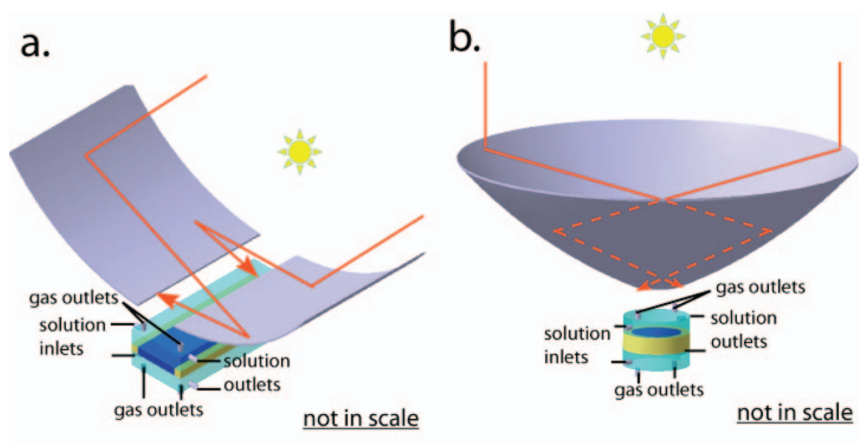

C.

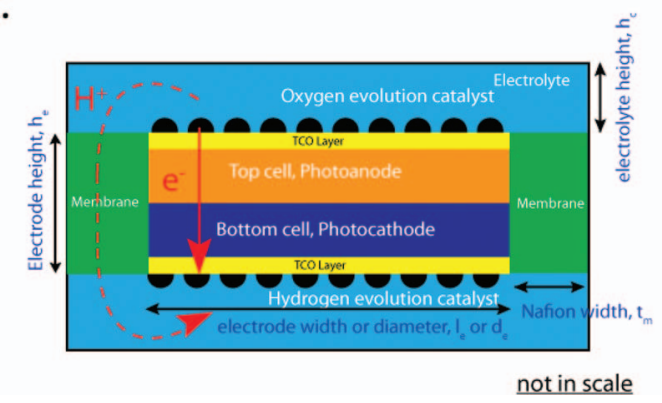

Figure 1. Schematic illustrations of two designs for a $10 \times$ concentrator photoelectrolysis system: a) a two-dimensional "trough" design and b) a threedimensional "bubble wrap" design. Both designs contain a solar concentrator (gray) and an integrated photoelectrolysis assembly. The orange lines schematically indicate selected ray traces of the light path that concentrates the sunlight. c) Cross-sectional illustration of an integrated photoelectrolysis system, which includes a photoanode (orange), a photocathode (purple), TCO layers (yellow), oxygen-evolution catalysts (top black hemispheres), hydrogen-evolution catalysts (bottom black hemispheres), Nafion films (green) and solution electrolyte (blue). The electronically conductive pathways occur from the oxygenevolution catalysts through the TCO, the top cell, the bottom cell, and the second TCO, to the hydrogen-evolution catalysts (solid arrow). The main ionically conductive pathways (dashed arrows), that produce the predominant ohmic drop in the system, occur laterally from the oxygen-evolution catalysts (that are the site of proton production during current flow) in the top electrolyte to and through the surrounding membrane, and laterally in the bottom electrolyte to the hydrogen-evolution catalysts (that are the sites of proton consumption during current flow). The electrode width, electrode diameter, electrode height, electrolyte height and Nafion width are represented by $l_{e}, d_{e}$, $h_{e}, h_{c}, t_{m}$, respectively. The Nafion fractional areal coverage of the entire cell is represented by $\xi$. sign (Fig. 1a) contains a photoelectrode having a width, $l_{e}$, with $l_{e}$ constrained to be $10 \%$ of the total cell width, $l_{c}$, consistent with $10 \mathrm{x}$ concentrating optics. The photoelectrode was taken to be infinitely long in the out-of-plane direction. The three-dimensional "bubble wrap" design (Fig. 1b) consists of a disk photoelectrode with a diameter, $d_{e}$, that was constrained to be $32 \%$ of the cell diameter, $d_{c}$, again consistent with 10x concentrating optics. Both designs contained Nafion films and insulating plastics to prevent product crossover and for structural support. The Nafion films also provided the required pathways for ionic conduction between the photocathode and photoanode chambers. The detailed construct of the integrated photoelectrochemical cell is shown in Figure 1c. The photoanode and photocathode are each assumed to be coated by a protective transparent conductive oxide (TCO) layer, to aid in uniformity of the current density distribution and minimize the ohmic losses in the system. Oxygen-evolution catalysts were coated on top of the TCO in the photocathode, whereas hydrogen-evolution catalysts were coated on the bottom of the TCO in the photoanode.

Table I presents the basic input parameters used in the model. The system performance was evaluated for two pairs of OER and HER catalysts in $1.0 \mathrm{M} \mathrm{H}_{2} \mathrm{SO}_{4}$. The "No. 1" catalyst system assumed electrocatalytic properties typical of iridium oxide for the OER and platinum for the HER in acidic conditions, while the "No. 2" catalyst system assumed twice the OER and HER overpotentials exhibited by the "No. 1" catalyst system to produce a current density of $100 \mathrm{~mA}$ $\mathrm{cm}^{-2}$ for the anodic and cathodic processes respectively, based on the geometric area of a planar electrode.

Equations describing transport and kinetics. - The ion transport in the electrolyte and in the proton-exchange membrane, as well as the transport of electrons and holes in the TCO coatings, were modeled using Ohm's law:

$$
j_{\mathrm{el}, \mathrm{TCO}, \mathrm{mem}}=-\sigma_{\mathrm{el}, \mathrm{TCO}, \mathrm{mem}} \nabla \phi_{\mathrm{el}, \mathrm{TCO}, \mathrm{mem}},
$$

where $\sigma_{e l, T C O \text {,mem }}$ is the conductivity of the electrolyte, TCO or the membrane (Nafion), respectively, $\nabla \phi_{e l, T C O, \text { mem }}$ is corresponding potential drop and $j_{e l, T C O, m e m}$ is the corresponding current density.

The kinetics of electrocatalysis at the TCO and at the electrolyte interface for the OER and HER were modeled using the Butler-Volmer equation:

$$
\begin{aligned}
j_{R, O E R / H E R}= & j_{0, O E R / H E R}\left[\exp \left(\frac{\alpha_{a, O E R / H E R} F \eta}{R T}\right)\right. \\
& \left.-\exp \left(-\frac{\alpha_{c, O E R / H E R} F \eta}{R T}\right)\right]
\end{aligned}
$$

where $j_{\mathrm{R}, O E R / H E R}$ is the current density for the OER or HER at the electrode/electrolyte interface, $j_{0, O E R / H E R}$ is the exchange-current density for the OER or HER, respectively, $\alpha_{a, O E R / H E R}$ and $\alpha_{c, O E R / H E R}$ are the corresponding anodic and cathodic transfer coefficients, respectively, $F$ is Faraday's constant, $R$ is the ideal gas constant, $T$ is the temperature and $\eta$ is the overpotential for the reaction, which is defined as

$$
\eta=\phi_{s}-\phi_{l}-\phi_{0}
$$

where $\phi_{s}$ and $\phi_{l}$ are the electric and electrolyte potentials at the interface, respectively, and $\phi_{0}$ is the equilibrium potential for the reaction. Conservation of charge and continuity of current density were enforced at both the cathodic and anodic interfaces.

Photoelectrode and temperature dependence.- The photoelectrode was modeled as two semiconducting light absorbers connected in series. One absorber had a bandgap of $1.7 \mathrm{eV}$ and was located directly on top of the other absorber, which had a bandgap of $1.1 \mathrm{eV}$. Without solar concentration, such a tandem cell can provide sufficient photovoltage at light-limited current densities to allow the use of earth-abundant catalyst materials in a photoelectrolysis system. ${ }^{18}$ The STH conversion efficiency as a function of different bandgap combinations with realistic cell geometries was also calculated in this study. 
Table I. Basic parameters used in the model.

Operating temperature

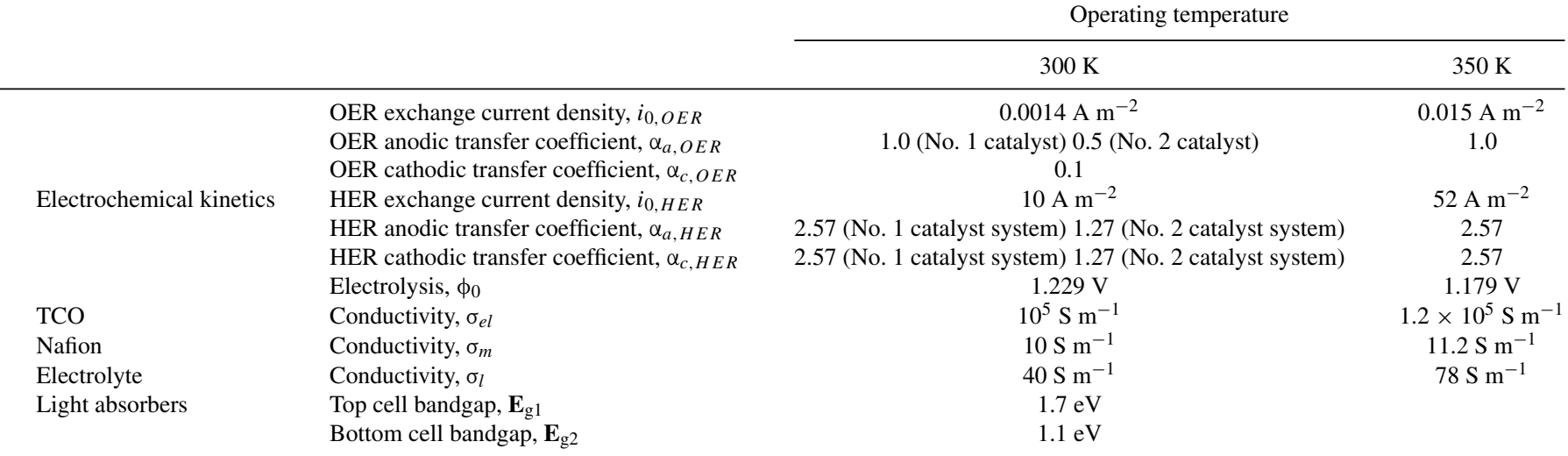

The photovoltage of the tandem photoelectrode at a given operating current density, $J$, was determined by adding the photovoltages of the serially connected top and bottom half-cells, i.e.:

$$
V_{\text {tandem }}(J)=V_{\text {top }}(J)+V_{\text {bottom }}(J) .
$$

The relationship between the current density and voltage of the top and bottom half-cells was determined using a detailed-balance calculation, in which the current density of each half-cell was set equal to the sum of the current density produced by the incident solar radiation $\left(J_{p h}\right)$ and the thermal radiation $\left(J_{t h}\right)$, minus the current density from radiative emission $\left(J_{r a d}\right)$ :

$$
J=J_{p h}+J_{t h}-J_{\text {rad }} .
$$

The analytical expressions for $J_{p h}, J_{t h}$ and $J_{r a d}$ are well described in literature. ${ }^{24,25}$ The operating current density of each half-cell was then calculated numerically at a given photovoltage. $100 \%$ of the above-bandgap solar flux was assumed to be absorbed and converted to photocurrent, so the photocurrent density was given by:

$$
J_{p h}=C \times e \int_{\mathrm{E}_{g}}^{\infty} \mathrm{d} \hbar \omega \frac{\Lambda}{\mathrm{d} \hbar \omega},
$$

where $C$ is the concentration factor, $e$ is the unsigned charge on an electron, $\mathbf{E}_{\mathrm{g}}$ is the bandgap of the photoabsorber, $\Lambda$ is the wavelengthdependent solar flux in the Air Mass (AM)1.5 solar spectrum, $\hbar$ is an abbreviation for $h / 2 \pi$ with $h$ being Planck's constant, and $\omega$ is the frequency of the incident light.

The radiative-emission currents were calculated from the relationship:

$$
J_{\text {rad }}=\frac{e\left(n_{\text {top }}^{2}+n_{\text {bottom }}^{2}\right)}{4 \pi^{2} c^{2}} \int_{\mathbf{E}_{g} / \hbar}^{\infty} \omega^{2} \exp \left(\frac{e V-\hbar \omega}{k T}\right) \mathrm{d} \omega,
$$

where $n_{\text {top }}$ and $n_{\text {bottom }}$ are the refractive indexes of the media on the top and at the bottom of the cell, respectively, $V$ is the operating voltage, $T$ is the absolute temperature, $k$ is Boltzmann's constant, and $c$ is the speed of light. The thermal radiation currents as a function of $T$ were given by:

$$
J_{t h}=\frac{e\left(n_{\text {top }}^{2}+n_{\text {bottom }}^{2}\right)}{4 \pi^{2} c^{2}} \int_{\mathbf{E}_{g} / \hbar}^{\infty} \omega^{2} \exp \left(-\frac{\hbar \omega}{k T}\right) \mathrm{d} \omega .
$$

The numerical relationship between the current-density and voltage that was obtained from the detailed-balance calculation was then fitted using eq. 9 for an ideal diode coupled with an effective series resistance:

$$
J=J_{p h}-J_{0}\left\{\exp \left[\frac{e\left(V+J R_{s}\right)}{k T}\right]-1\right\} .
$$

where $J_{0}$ is saturation current density in the dark, $V$ is the applied photovoltage, and $R_{\mathrm{S}}$ is the effective series resistance. The fitted value for $J$ was then used as the boundary condition at the photoelectrode-TCO

interface. The effective series resistance was varied to approximate various situations in which the solar cell operated either at the detailedbalance limit or under non-ideal operating conditions in which the fill factor varied from 0.65 to 0.90 .

Temperature-dependent conductivity of the electrolyte and membrane.- The conductivity of the electrolyte as a function of the operating temperature was defined by:

$$
\sigma_{l, T}=\sigma_{l, r e f}\left(1+\alpha\left[T-T_{r e f}\right]\right),
$$

where the reference temperature, $T_{r e f}$, was $300 \mathrm{~K}$ and the coefficient $\alpha$ was $0.019 \mathrm{~K}^{-1}$, which was fitted using experimental data for $1 \mathrm{M}$ sulfuric acid. ${ }^{26}$

The temperature-dependent conductivity of the Nafion and the TCO layer was calculated by:

$$
\begin{aligned}
\sigma_{m e m / T C O, T}= & \sigma_{m e m / T C O, T_{r e f}} \exp \left(\frac{E_{a, m e m / T C O}}{R T_{r e f}}\right) \\
& \times \exp \left(-\frac{E_{a, m e m / T C O}}{R T}\right),
\end{aligned}
$$

where $\sigma_{m e m / T C O} T_{r e f}$ is the Nafion/TCO conductivity at the reference temperature $(300 \mathrm{~K}), E_{a, \mathrm{mem} / \mathrm{TCO}}$ is the activation energy for Nafion (set to $2000 \mathrm{~J} \mathrm{~mol}^{-1}$ ) 27,28 and for TCO (set to $3185 \mathrm{~J} \mathrm{~mol}^{-1}$ ) ${ }^{29}$, respectively. The exchange-current density for the OER and HER, respectively, in the Butler-Volmer equation was defined using the activation energy $E_{\text {a.OER/HER }}:^{30}$

$$
\begin{aligned}
j_{0, T, O E R / H E R}= & j_{0, T_{r e f}, O E R / H E R} \exp \left(\frac{E_{a, O E R / H E R}}{R T_{r e f}}\right) \\
& \times \exp \left(-\frac{E_{a, O E R / H E R}}{R T}\right),
\end{aligned}
$$

where $j_{0, T_{\text {ref }}, O E R / H E R}$ is the exchange current density for the OER or HER at the reference temperature, and $E_{a, O E R / H E R}$ was assumed to be $42560 \mathrm{~J} \mathrm{~mol}^{-1}$ and $28900 \mathrm{~J} \mathrm{~mol}^{-131,32}$ for iridium oxide ${ }^{33}$ and platinum ${ }^{24,25}$ catalysts, respectively, in $1 \mathrm{M} \mathrm{H}_{2} \mathrm{SO}_{4}$ (aq).

Boundary conditions. - Boundary conditions that preserved the symmetry of the current density at the vertical walls were employed for both the trough and bubble wrap designs. Insulating boundary conditions were used at the upper and bottom bounds of the electrolyte domains. The current flux entering the TCO layers was calculated from the photovoltage using eq. 9. The electrochemical reactions for both designs were modeled as surface reactions occurring at the interface of the solution and the TCO layer. The ionic conductivities of the Nafion film and of $1 \mathrm{M} \mathrm{H}_{2} \mathrm{SO}_{4}$ that were used in the modeling are listed in Table I. For the crossover calculation, the Dirichlet (or first-type) boundary condition was employed at the interfaces of the electrolyte and the TCO layers. The hydrogen (oxygen) concentrations at the 
cathode (anode) were set to $0.78 \mathrm{mM}(0 \mathrm{mM})$ and $0 \mathrm{mM}(1.23 \mathrm{mM})$, respectively. Insulating boundary conditions for the product gas flux were also applied at the upper and bottom bounds of the solution domains.

Free triangular discretization and a standard solver in the Comsol multi-physics package were used in the modeling. For both designs, the maximum and minimum mesh-element sizes were set to $10 \mu \mathrm{m}$ and $0.5 \mu \mathrm{m}$, respectively. The maximum mesh-element growth rate and the resolution of curvature were set at 1.3 and 0.3 , respectively. A relative tolerance of the corresponding variable of 0.001 was applied as the convergence criterion for both designs.

Operating principles of device efficiency. - The instantaneous STH conversion efficiency of an integrated photoelectrolysis device, $\eta_{S T H}$, was defined as:

$$
\eta_{S T H}=\frac{J \cdot \phi_{0}}{P} \eta_{F} \eta_{p c}
$$

where $J$ is the current density output from the device, $\phi_{0}$ is the equilibrium potential of the electrochemical reaction, $P$ is the input power of the solar energy, $\eta_{F}$ is the faradaic efficiency of the electrode reaction, and $\eta_{p c}$ is the product-collection efficiency. $\eta_{F}$ was set to unity in the model, while $\eta_{p c}$ was defined by the following equation:

$$
\eta_{p c}=\frac{\int j d A-\int_{A_{a / c}} n F N_{\text {fuel }} d A}{\int_{A_{a / c}} j d A},
$$

where $j$ is the current density at the interfaces between the catalysts and the solution, the net reaction current, $N_{f u e l}$, represents the current lost due to diffusive crossover of hydrogen from the cathode to the anode chamber, $n$ is number of electrons transferred ( 2 for the HER and 4 for the OER), $A_{a / c}$ and $A_{\text {sep }}$ are the electrode area and the Nafion area that separates the anodic and cathodic chambers, respectively.

To obtain a simple 0 -dimensional analytical picture of the cell performance for diagnostic purposes, and therefore to aid in understanding the efficiency of the entire system at the individual-component level, the current-voltage characteristics of the cell, and the polarization curve for the water-splitting reaction, were typically overlaid to determine the operating current density.

Comparison to photovoltaic cell in series with an electrolyzer.The STH efficiency of a stand-alone photovoltaic cell connected electrically in series with a discrete electrolyzer (PV + electrolyzer) was defined as,

$$
\eta_{S T H}=\eta_{P V} \cdot \eta_{\text {electrolyzer }} \cdot \eta_{D C-D C-\text { converter }},
$$

where $\eta_{\mathrm{PV}}$ is the solar-to-electricity conversion efficiency of the photovoltaic cell, $\eta_{\text {electrolyzer }}$ is the electricity-to-hydrogen conversion efficiency of the electrolyzer and $\eta_{D C-D C \text {-converter }}$ is the efficiency of a DC-to-DC converter. The efficiency of the electrolyzer used for this comparison was the value optimized at a current density of $\sim 1 \mathrm{~A} \mathrm{~cm}^{-2}$ and within the temperature range from $70^{\circ} \mathrm{C}$ to $80^{\circ} \mathrm{C}$, representive of the performance characteristics of state-of-the-art commercially available electrolyzers. ${ }^{34-37}$ In this study, an electrolyzer efficiency of $73.0 \%$ and a DC-to-DC converter efficiency of $85 \%$ (representative of values for converters that need to perform dynamic maximum power point tracking on the input DC power from the PV array as well as independent tracking on the output DC power that is supplied to the electrolyzer to insure maximum electrolyzer efficiency) were used in the calculation. The efficiency of the PV was chosen to be the detailed balance limit, for the same tandem photoabsorber system with the same bandgap combination.

\section{Results}

Spatially non-uniform current density and catalytic overpotentials. - Figure 2 illustrates the results from a 0-dimensional analytical calculation of the performance of solar cells with three

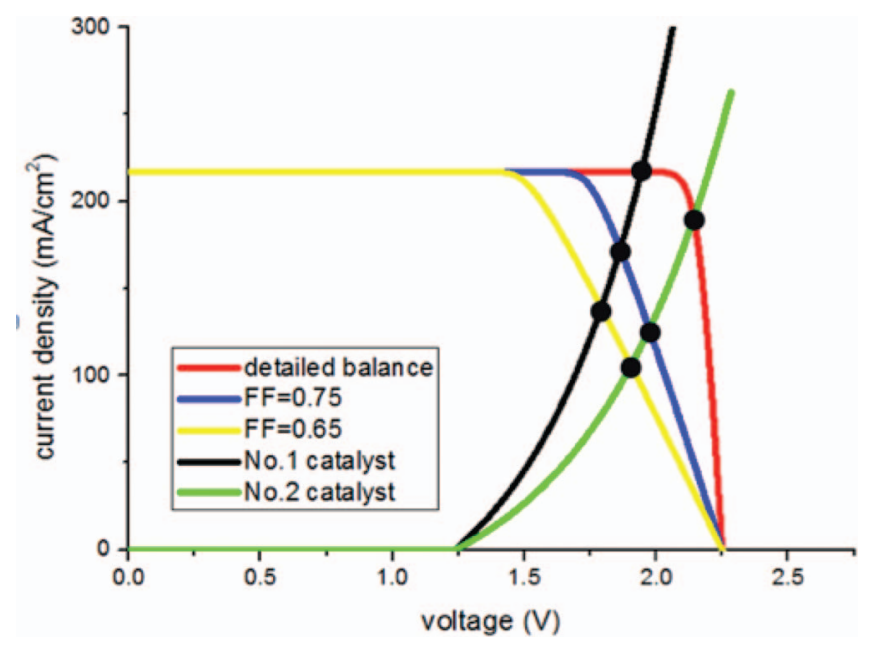

Figure 2. 0-dimensional calculations of the operating current densities determined by the crossing points between the water-splitting polarization curves and the current density-voltage characteristics for tandem photoelectrode materials with three different fill factors and two sets of catalysts. The detailed photovoltaic parameters and electrochemical parameters are listed in Table I.

fill factors: detailed balance, 0.75 , and 0.65 , and the accompanying polarization behavior for the two modeled catalyst systems. The operating temperature for the cells was $300 \mathrm{~K}$. The overall cell efficiency, which is determined by the operating current density at the crossing point, clearly depended strongly upon the combination of the current-voltage characteristics and the polarization behavior. However, the over-simplified 0-dimensional model cannot adequately describe the non-uniform distribution of current at the photoelectrode and the resistive loss in the solution associated with specific geometries, both of which could have significant impacts on the cell efficiency.

Figure 3 a shows the potential profile within the electrolyte and the current-density distribution for a cross section of a "trough" cell. Due to the spatial distribution of the solution resistance, the overall polarization behavior at different locations along the electrode width varied significantly, especially for large electrode widths. Thus, even with the same current-voltage performance of the light absorber, the crossing point of the water-splitting polarization behavior and the photodiode behavior, which determined the operating current density, varied along the electrode width. Consequently, the overpotentials for HER and OER along the electrode width also exhibited a strong position dependence. For example, Figure $3 \mathrm{~b}$ shows the OER overpotential along the electrode for electrode widths of $10 \mu \mathrm{m}, 1 \mathrm{~mm}, 3 \mathrm{~mm}$ and $5 \mathrm{~mm}$, respectively. The distribution of the current density along the electrode width was highly non-uniform for this light-concentrating photoelectrolysis system. Compared to an unconcentrated system (data not shown), the OER overpotential difference between the mid-point and the two ends of the electrode increased from $6.5 \mathrm{mV}$ to $49.4 \mathrm{mV}$ (Figure $3 \mathrm{~b}$ ) and the maximum resistive loss increased from $17.2 \mathrm{mV}$ to $143.1 \mathrm{mV}$ (Figure 3a) with the same electrode width, electrode height and Nafion area (note that the areal Nafion coverage for the un-concentrated system is 10 times larger than for the system that utilizes solar concentration).

STH conversion efficiency.- The performance as a function of three important system-related geometric parameters: the electrode width or diameter; the electrode height; and the areal Nafion coverage of the entire cell, was evaluated systematically for both types of cell designs. The solution height was set to $5 \mathrm{~mm}$ (from the electrode to the upper or lower bound of the cell) so that the cell performance had a very weak dependence on the solution height. ${ }^{17}$ The detailed current density versus voltage characteristic of the tandem photoabsorbers was expected to have a significant impact on the overall value of $\eta_{\mathrm{STH}}$. 


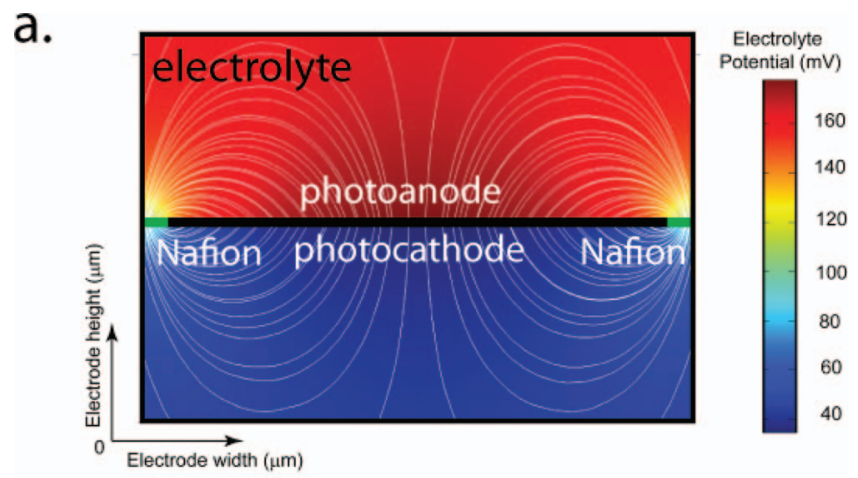

b.

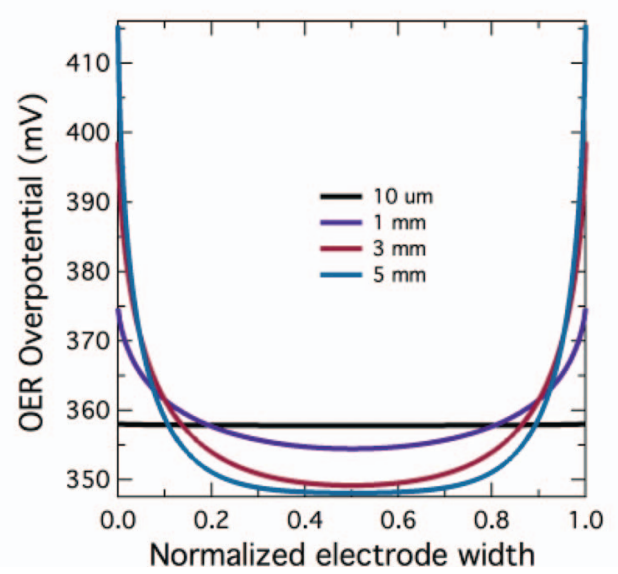

Figure 3. (a) Electrolyte potential-distribution profile for a "trough" design, with the electrode width, electrode height, solution height and Nafion coverage set to $3 \mathrm{~mm}, 10 \mu \mathrm{m}, 5 \mathrm{~mm}$ and $1 \%$, respectively. The black line represents the photocathode and photoanode assembly and the red lines represent the Nafion film. The streamlines represent the electrolyte current density. (b) The OER overpotential as a function of the normalized electrode width for a "trough" design with four electrode widths: $10 \mu \mathrm{m}$ (black), $1 \mathrm{~mm}$ (red), $3 \mathrm{~mm}$ (green) and $5 \mathrm{~mm}$ (blue) under detailed-balance conditions at $350 \mathrm{~K}$.

Figure 4 shows $\eta_{\text {STH }}$ for the trough (a) and the bubble wrap (b) designs as a function of the electrode width, the electrode height and the Nafion coverage, for light absorbers operating at fill factors of 0.65 (i), 0.75 (ii), and for the detailed-balance limit (iii), respectively, at $350 \mathrm{~K}$. In both designs, $\eta_{\mathrm{STH}}$ increased monotonically as the electrode width and height decreased, and as the Nafion coverage increased. The value of $\eta_{\mathrm{STH}}$ also increased as the fill factor of the tandem light absorbers increased from 0.65 to 0.885 (detailed-balance condition) in all cases. While the two designs showed similar efficiency trends, the efficiency of the bubble-wrap design was slightly higher than that of the trough design, when the electrode width of the trough design was equal to the electrode diameter in the bubble-wrap design. This difference occurred due to enhanced radial transport in the electrolyte in the latter design. For small electrode dimensions, the cell efficiencies exhibited a weak dependence on the Nafion coverage. For example, in the bubble-wrap design, increasing the Nafion coverage from $0.2 \%$ to $1 \%$ resulted in an increase in $\eta_{\mathrm{STH}}$ of a large electrode $\left(d_{e}=1\right.$ $\left.\mathrm{cm}, h_{e}=100 \mu \mathrm{m}\right)$ from $11.7 \%$ to $25.0 \%$, but for small electrode $\left(d_{e}=10 \mu \mathrm{m}, h_{e}=1 \mu \mathrm{m}\right) \eta_{\mathrm{STH}}$ remained unchanged at $25.5 \%$. The value of $\eta_{\mathrm{STH}}$ reached a plateau as the Nafion coverage reached $1 \%$ of the entire cell width for both designs, which indicated that a large portion of the separator could be made of non-porous plastic without compromising the cell performance in either design. The decrease of the STH conversion efficiency due to the product gas crossover in both designs, even with smallest cell dimensions, was negligible (< $0.5 \%$ ) due to the use of a membrane separator.
Ohmic losses associated with proton transport in the solution and in the membrane separator-- An efficient solar-to-fuel generator requires low $(<100 \mathrm{mV})$ ohmic losses in the entire system. ${ }^{17}$ The ohmic loss is however highly dependent on the cell geometry. As shown in Figure 1c, both electrons and ions must be transported at steadystate from the anode/OER interface to the cathode/HER interface. Hence, the electronically conductive pathways occur from the oxygenevolution catalysts through the TCO, the top cell, the bottom cell, and the second TCO, to the hydrogen-evolution catalysts. In contrast, the main ionically conductive pathways, that produce the predominant ohmic drop in the system, occur laterally from the oxygen-evolution catalysts (that are the site of proton production during current flow) in the top electrolyte to and through the surrounding membrane, and laterally in the bottom electrolyte to the hydrogen-evolution catalysts (that are the sites of proton consumption during current flow). Figures $5 \mathrm{a}$ and $5 \mathrm{~b}$, respectively show the ohmic loss calculated using system parameters that correspond to Figures 4(a) (iii) and 4(b) (iii), respectively. To produce low ohmic losses in the trough design, the electrode width cannot exceed $2 \mathrm{~mm}$, and the electrode height cannot exceed 40 $\mu \mathrm{m}$ (Figure 5a). The corresponding low ohmic loss criterion for the bubble-wrap design was satisfied when the electrode diameter did not exceed $3 \mathrm{~mm}$ and the electrode height did not exceed $50 \mu \mathrm{m}$ (Figure $5 \mathrm{~b})$. Compared to the trough design, the same electrode dimensions in the bubble-wrap design resulted in less resistive loss due to improved radial transport of ionic species in the electrolyte. Interestingly, a very small Nafion coverage $(0.2 \%)$ could produce an ohmic resistive loss of $<100 \mathrm{mV}$ in a bubble-wrap cell with an electrode diameter as large as $2 \mathrm{~mm}$.

Effect of catalysts. - The $10 \times$ concentrator design will operate at a higher current density ( $\sim 10 \times$ higher on average) than an unconcentrated system, so the kinetic overpotentials for HER and OER may be more critical to the overall $\eta_{\mathrm{STH}}$ in concentrated designs than in systems that do not utilize solar concentration. Figure 6 compares $\eta_{\text {STH }}$ for both pairs of catalysts under detailed-balance conditions at $300 \mathrm{~K}$. For small electrode sizes (electrode width $<600 \mu \mathrm{m}$ and electrode height $<10 \mu \mathrm{m}$ for the trough design or electrode diameter $<1$ $\mathrm{mm}$ and electrode height $<20 \mu \mathrm{m}$ for the bubble-wrap design) $\eta_{\mathrm{STH}}$ was essentially unchanged ( $<3$ percentage-point difference) when the different electrocatalysts were used, because the water-splitting polarization and photodiode behavior crossed at the plateau of the current density versus voltage curve, where the operating current density remained nearly constant. As the electrode width increased, $\eta_{\mathrm{STH}}$ exhibited a stronger geometric dependence on the properties of the electrocatalysts. For instance, for the large electrode $\left(l_{e}=1 \mathrm{~cm}\right.$, $h_{e}=1 \mu \mathrm{m}$ and $\xi=0.2 \%$ ) in the trough design, $\eta_{\mathrm{STH}}$ decreased from $22.1 \%$ to $7.3 \%$ when the "No. 1" catalyst pair was exchanged for the "No. 2" catalyst pair. The 0-dimensional analysis in Figure 2 illustrated the situation in which $\eta_{\text {STH }}$ was strongly dependent on the catalyst activities at large electrode dimensions.

Effect of operating temperature.- Figure 7 shows $\eta_{\mathrm{STH}}$ for the systems at operating temperatures of $300 \mathrm{~K}$ and $350 \mathrm{~K}$, respectively, with various combinations of the geometric parameters and with the photoabsorbers assumed to operate at the Shockley-Queisser detailedbalance limit. Two geometric regions, that exhibited opposite dependences of $\eta_{\text {STH }}$ vs $T$, were observed in the simulations. Figures 7 a (iii) and $7 \mathrm{~b}$ (iii) show, for both designs, the difference between $\eta_{\mathrm{STH}}$ at $350 \mathrm{~K}$ and $\eta_{\mathrm{STH}}$ at $300 \mathrm{~K}$. When the solution transport and kinetic overpotential losses were small (i.e. at small electrode dimensions, and with a large Nafion coverage), $\eta_{\text {STH }}$ decreased with increased operating temperature. For example, $\eta_{\mathrm{STH}}$ decreased from $26.7 \%$ to $25.5 \%$ for an electrode that was $10 \mu \mathrm{m}$ wide and $1 \mu \mathrm{m}$ thick when the Nafion coverage was $1 \%$ in the trough design (and from $26.7 \%$ to $25.5 \%$ for an electrode with a diameter of $10 \mu \mathrm{m}$ and $1 \mu \mathrm{m}$ thick when the Nafion coverage was $1 \%$ in the bubble-wrap design). In this geometric region, the decrease of $\eta_{\mathrm{STH}}$ with increasing $T$ was dominated by the degradation of the performance of the light absorbers as $T$ increased. In contrast, when the solution-transport loss and the kinetic 
a.

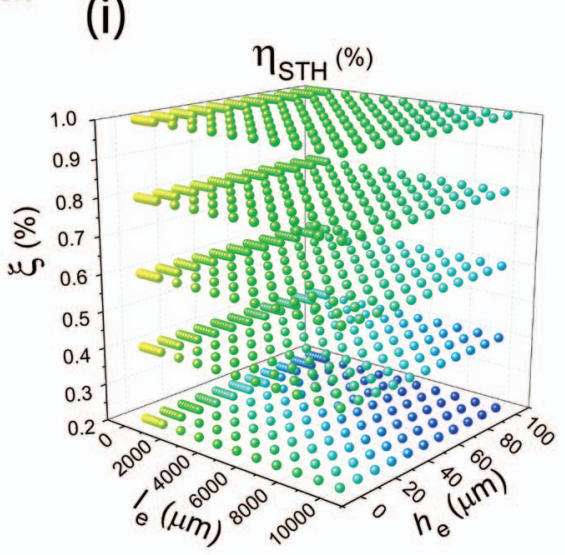

(ii)

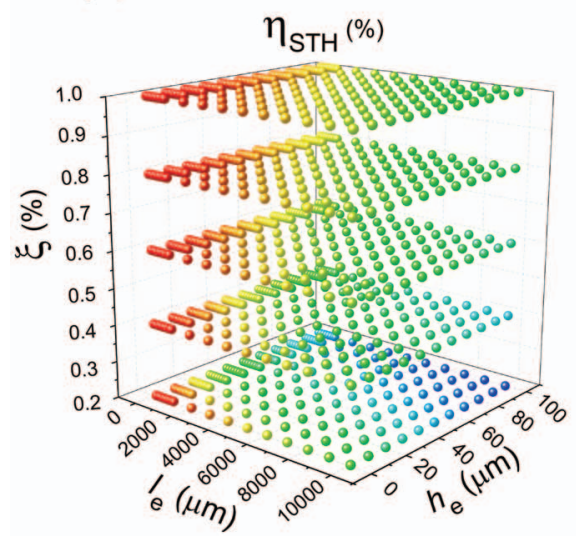

(iii)

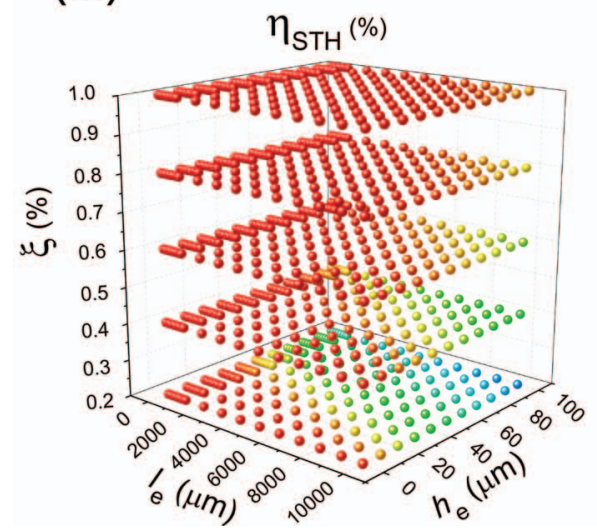

b.

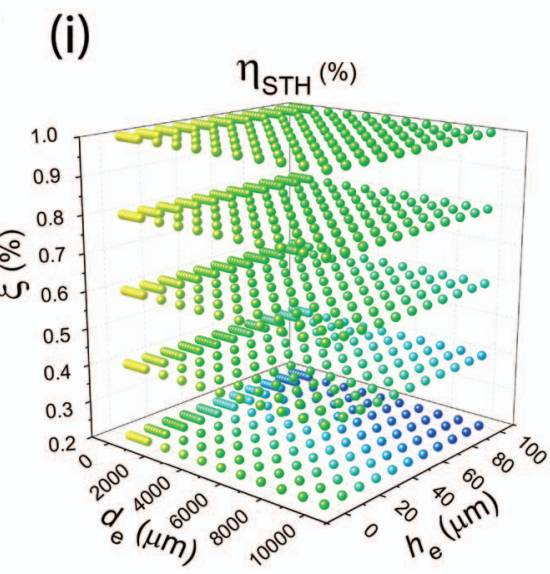

(ii)
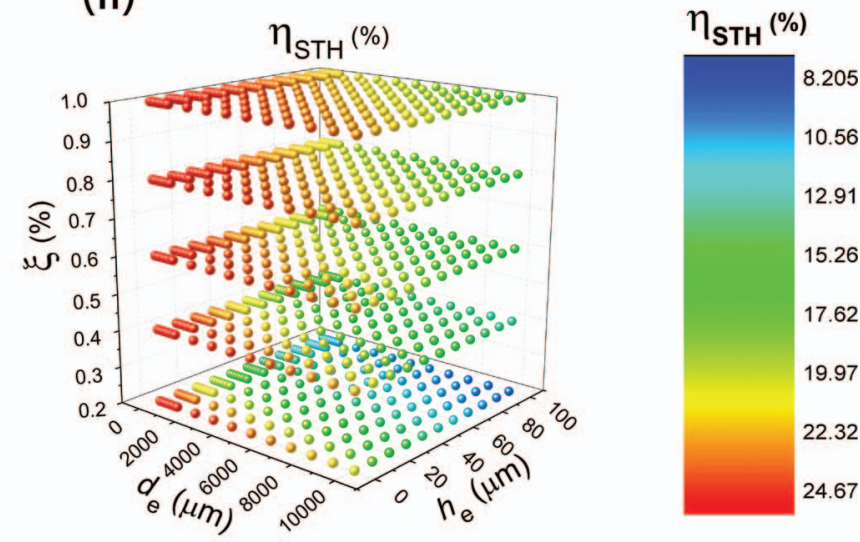

Figure 4. $\eta_{\mathrm{STH}}$ calculated for the "trough" design (a) and for the "bubble wrap" design (b) as a function of the electrode width/diameter, $l_{\mathrm{e}} / d_{\mathrm{e}}$, the electrode height, $\mathrm{h}_{\mathrm{e}}$, and the Nafion coverage, $\xi$, for the tandem photoabsorbers at $350 \mathrm{~K}$ with a fill factor of 0.65 (i), 0.75 (ii) and at the detailed-balance limit (iii). The electrode width/diameter, $l_{\mathrm{e}} / d_{\mathrm{e}}$, was varied from $10 \mu \mathrm{m}$ to $1 \mathrm{~mm}$, with an interval of $150 \mu \mathrm{m}$, as well as from $1 \mathrm{~mm}$ to $1 \mathrm{~cm}$, with an interval of $1 \mathrm{~mm}$. The electrode height, $h_{\mathrm{e}}$, was set from $1 \mu \mathrm{m}$ to $100 \mu \mathrm{m}$, with an interval of $10 \mu \mathrm{m}$. The Nafion coverage, $\xi$, was set from $0.2 \%$ to $1 \%$, with an interval of 0.2 .

overpotential losses were large (i.e. at large electrode dimensions and with a small Nafion coverage), $\eta_{\mathrm{STH}}$ increased as $T$ increased. For example, $\eta_{\mathrm{STH}}$ increased from $8.9 \%$ to $10.2 \%$ with a $1 \mathrm{~cm}$ electrode width, $100 \mu \mathrm{m}$ electrode height and $0.2 \%$ Nafion coverage for the trough design (and from $10.6 \%$ to $11.7 \%$ with $1 \mathrm{~cm}$ electrode diameter, $100 \mu \mathrm{m}$ electrode height and $0.2 \%$ Nafion coverage for the bubble-wrap design). In this geometric region, the increase of the $\eta_{\mathrm{STH}}$ was dominated by enhanced rate of electrocatalysis as well as by enhanced solution transport as the temperature increased.
Comparison to a solar-hydrogen generator without a solar concentrator.- While a significant reduction of materials usage in solar concentrator-coupled photoelectrochemical cells is advantageous compared to cells without solar concentration, higher operating current densities in the concentrator design would result in higher catalytic overpotentials and higher transport loss and thus would lower the STH conversion efficiency of the cell. Figure 8 shows $\eta_{\text {STH }}$ of an unconcentrated cell system and of the 10x "trough" design, with identical cell geometries for the tandem photoabsorbers, at different 
a.

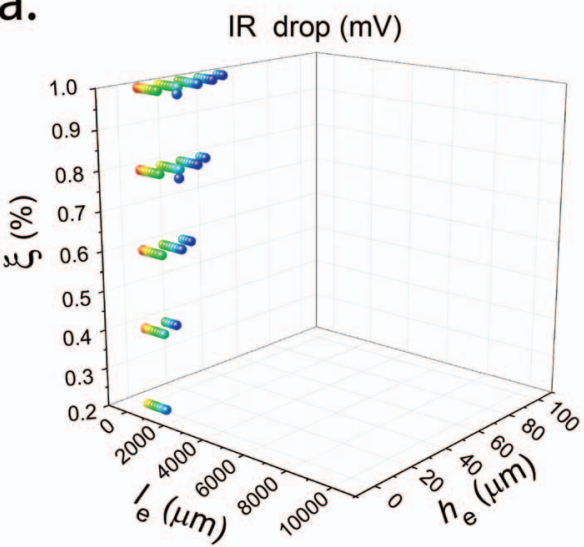

b.

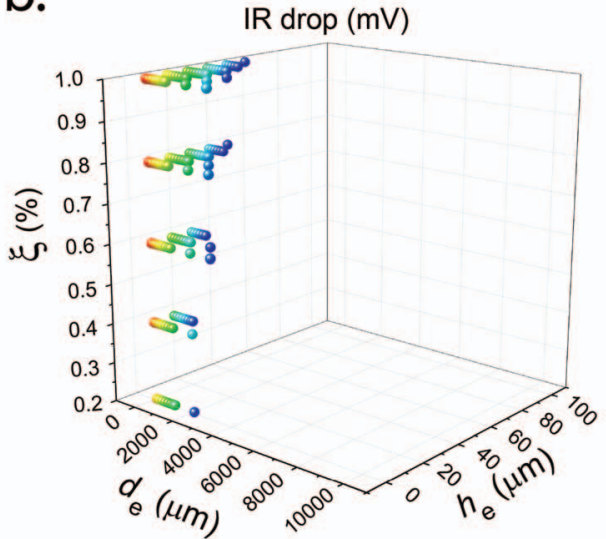

IR drop (mV)

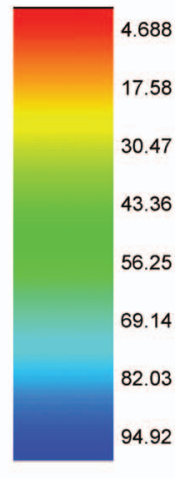

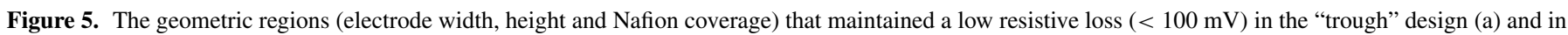
the "bubble wrap" design (b).

bandgap combinations. With the optimized cell geometry and active catalysts ("No. 1" catalyst system), $\eta_{\mathrm{STH}}$ exhibited little change between the $10 \times$ the concentrated and unconcentrated systems. However, when the cell geometry was not optimized, a significant decrease in $\eta_{\mathrm{STH}}$ and a significant difference in the bandgap combination dependence of the cell efficiency were observed. A similar change in $\eta_{\mathrm{STH}}$ as a function of the bandgap combination was also observed in the "bubble-wrap" design. When the state-of-the-art catalyst system was used and the cell geometry was optimized, $\eta_{\mathrm{STH}}$ in both the concentrated and unconcentrated systems were not limited by the electrocatalysis or the solution resistive loss. As a result, no significant difference in $\eta_{\mathrm{STH}}$ was found between the two systems. However, when the resistive loss or electrocatalysis became the limiting factor in the system, a notable change in $\eta_{\mathrm{STH}}$ was calculated between the a.

(i)

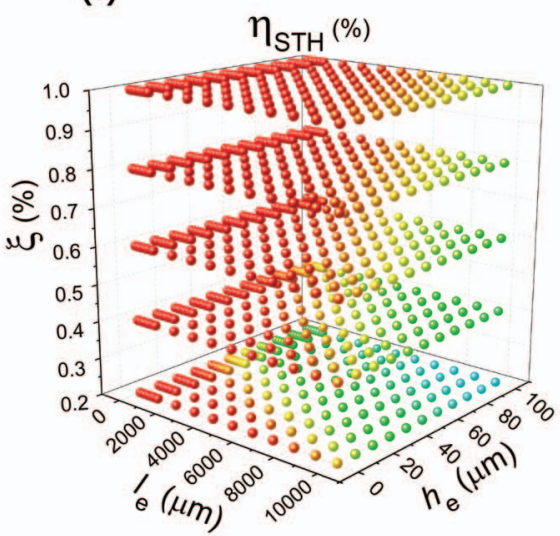

b. (i)

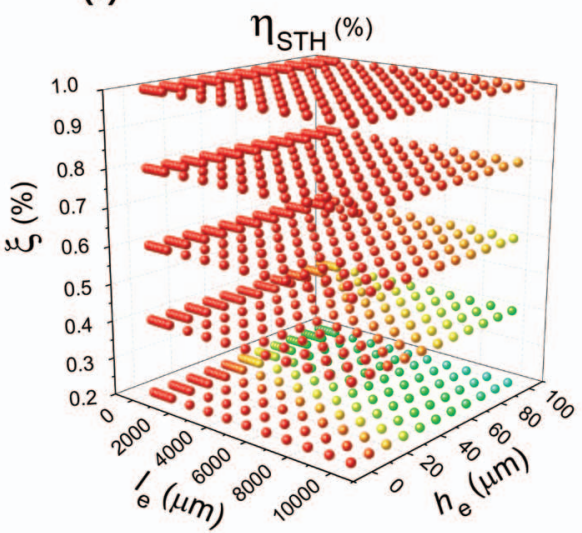

(ii)

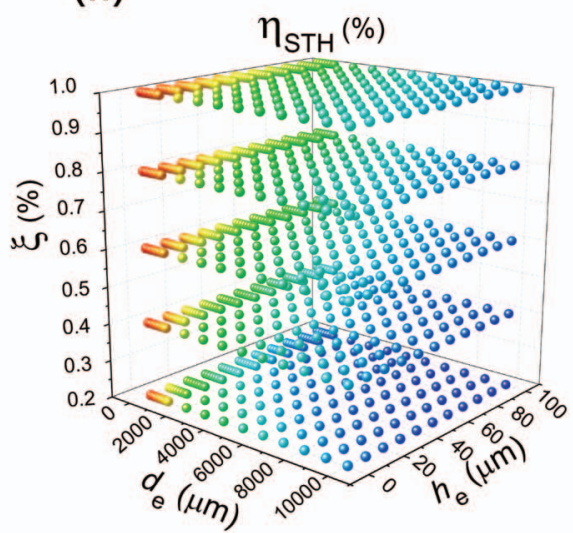

(ii)

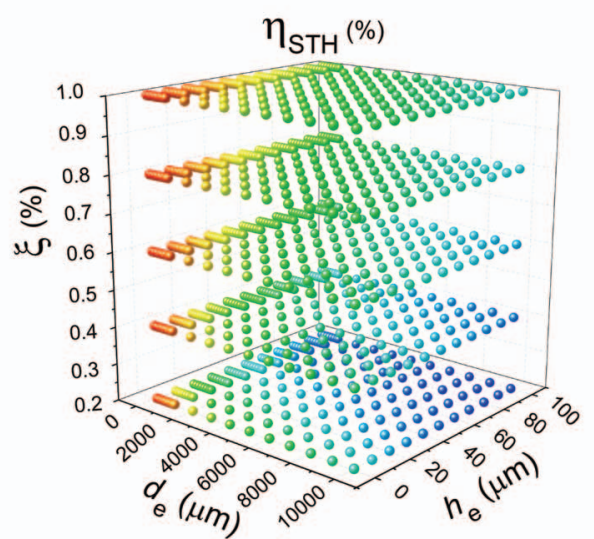

$\eta_{\text {STH }}(\%)$

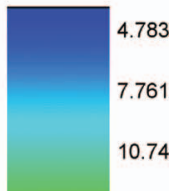

13.72

16.69

19.67

22.65

25.63

Figure 6. $\eta_{S T H}$ for the trough (a) and bubble-wrap (b) designs with the "No. 1" catalyst system (i) and the "No. 2" catalyst system (ii). 
a.

(i)

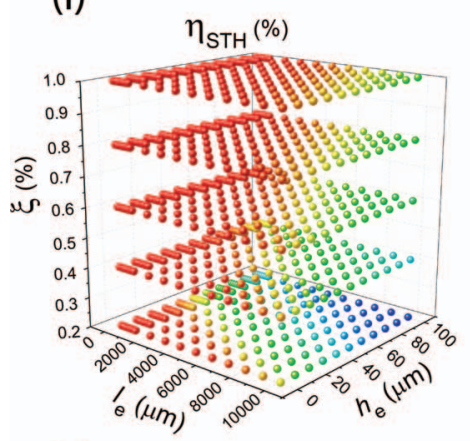

(ii)

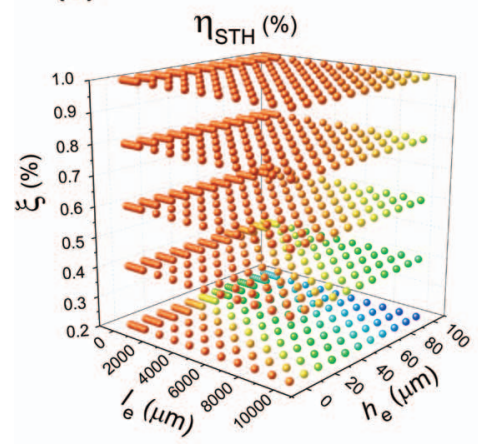

(iii)

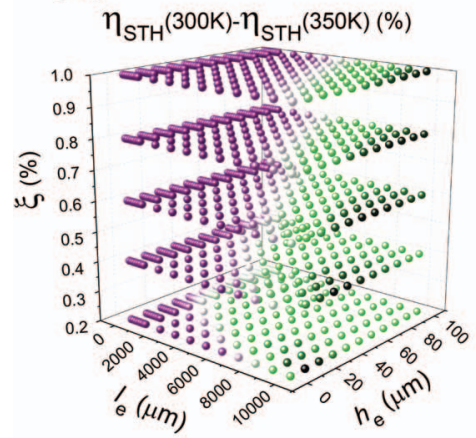

b.

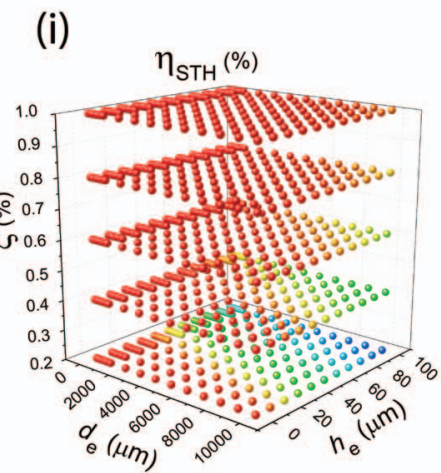

(ii)
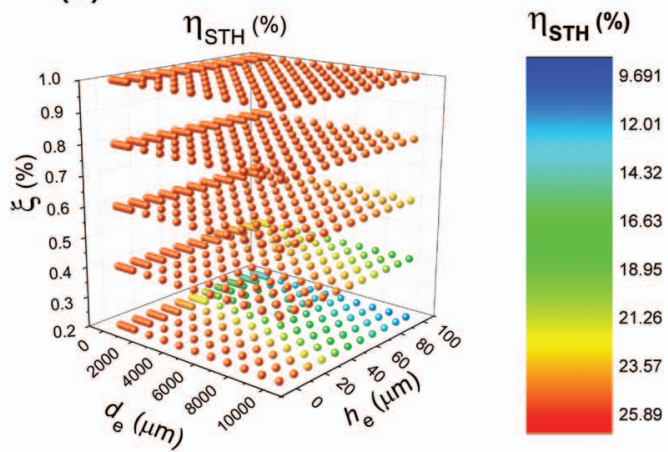

(iii)

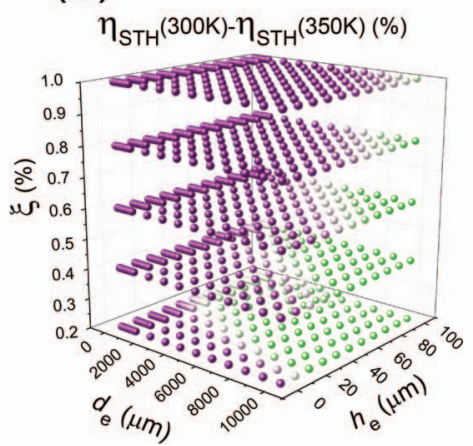

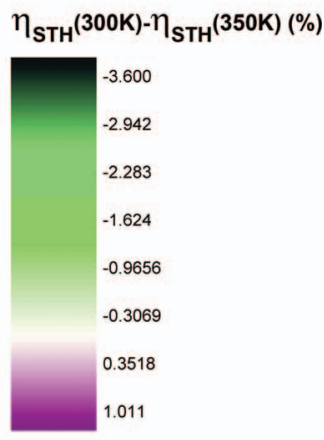

Figure 7. $\eta_{\mathrm{STH}}$ at $300 \mathrm{~K}$ (i) and $350 \mathrm{~K}$ (ii) and the STH conversion-efficiency difference in percentage points (iii) for the trough (a) and the bubble-wrap (b) designs.

two systems. From the simulation, the concentrated system exhibited a stronger dependence on the cell geometry than the unconcentrated system.

Comparison to a standalone PV + electrolyzer design.- Figure 8 shows $\eta_{\mathrm{STH}}$ of a stand-alone PV+electrolyzer system (e) and of the integrated 10x "trough" design with an optimal geometry (b) as a function of different bandgap combinations at $350 \mathrm{~K}$. In both systems, state-of-the-art catalysts ("No. 1" catalyst system) were assumed. The highest value of $\eta_{\mathrm{STH}}$ for the PV+electrolyzer system was $25.9 \%$, when the top material had a $1.6 \mathrm{eV}$ bandgap and the bottom material had a bandgap of $0.9 \mathrm{eV}$. For comparison, when the cell geometry was optimized, the integrated "trough" design exhibited $\eta_{\mathrm{STH}}$ $=29.8 \%$ at the same bandgap combination. A similar enhancement of $\eta_{\mathrm{STH}}$ was also observed in the "bubble-wrap" design relative to the PV+electrolyzer system. Of course, the efficiency values for the stand-alone PV+electrolyzer system are directly proportional to the values assumed for the dynamic DC-DC converter (85\%) as well as the efficiency of the electrolyzer (73\%), with changes in these subsystem efficiencies simply linearly affecting the overall efficiency values presented for the different bandgap combinations in Figure 8.

\section{Discussion}

The higher currents in the $10 \times$ concentrator system increased the ohmic loss and also stressed the catalyst performance. However, the modeling described herein revealed that optimized cell designs, with the critical dimension of the photoelectrodes being less than a few millimeters, can result in very high STH conversion efficiencies, with $\eta_{\mathrm{STH}}=29.8 \%$ (Fig. $8 \mathrm{~b}$ ). These $\eta_{\mathrm{STH}}$ values are almost identical to the optimized instantaneous $\eta_{\mathrm{STH}}$ values for an integrated PEC system at 1 Sun (Fig. 8a). These $10 \times$ concentrator designs should therefore be considered as viable alternatives to "flat plate" designs that have been evaluated previously which utilize unconcentrated sunlight.

The millimeter electrode length scale indicated by the modeling and simulation to be required for optimum $\eta_{\mathrm{STH}}$ with $10 \times$ solar concentration could be readily implemented in a practical electrode manufacturing and cell assembly process. Moreover, the reduction of the materials usage for the photoelectrode $(10 \%$ of the system area) and the Nafion ( $<1 \%$ of the system area) would significantly reduce the cost of these potentially expensive components of a whole, scalable, solar fuels generation system. Large areas of insulating plastic ( $\sim 90 \%$ of the system area) and the 
a.
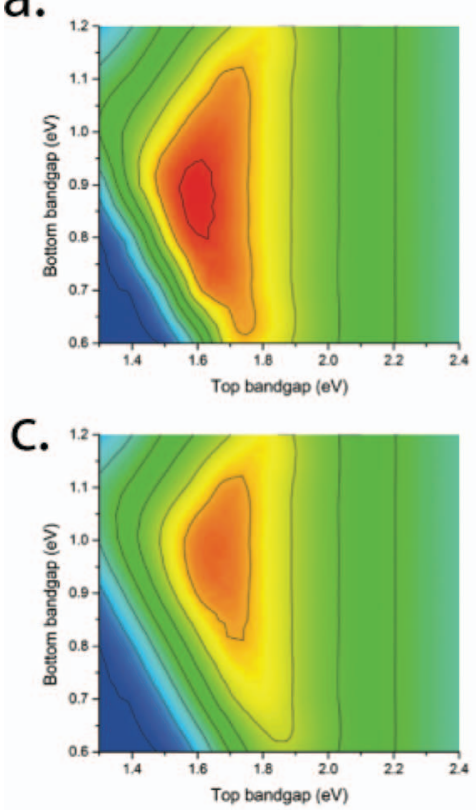

d.
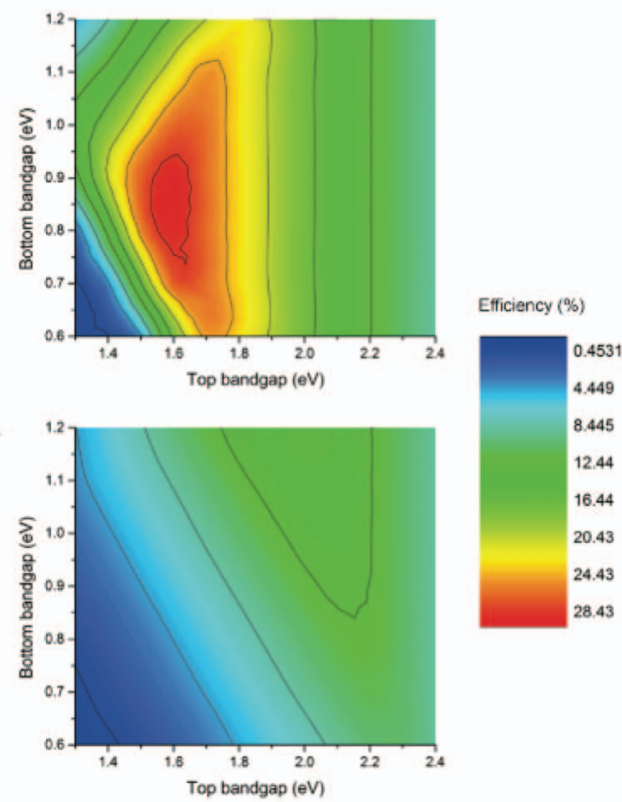

Figure 8. $\eta_{\mathrm{STH}}$ as a function of different bandgap combinations for an unconcentrated system with optimized cell geometry (a), the $10 \times$ "trough" design with optimized cell geometry (b), an unconcentrated system with non-optimized cell geometry $\left(l_{\mathrm{e}}=10\right.$ $\mu \mathrm{m}, h_{\mathrm{e}}=1 \mu \mathrm{m}, \xi=1 \%$ ) (c) and the $10 \times$ "trough" design with non-optimized cell geometry $\left(l_{\mathrm{e}}=1 \mathrm{~cm}\right.$ $h_{\mathrm{e}}=100 \mu \mathrm{m}, \xi=0.2 \%$ )(d) at $350 \mathrm{~K}$. $\eta_{\mathrm{STH}}$ as a function of different bandgap combinations for a standalone PV+electrolyzer system (e) at $350 \mathrm{~K}$. The "No. 1 catalyst system" was employed in all calculations.

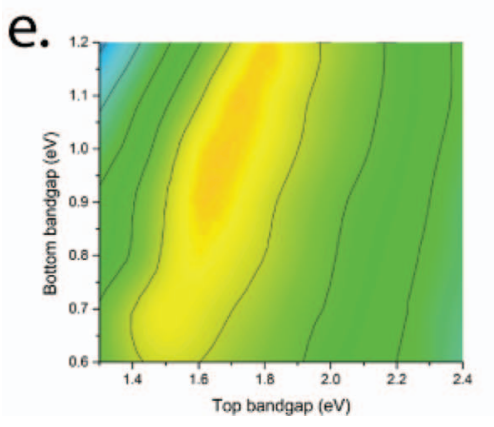

absence of a diurnal solar and system temperature regulating system would reduce the maintenance of the system and additionally would likely reduce the cost of the balance of systems in the solar fuels generator.

In operation, the system will likely be tilted at some angle relative to the surface normal. However, the light absorption, carrier transport, electrocatalysis and solution transport analyzed herein within the constraints of the present model are not expected to be sensitive to the tilt angle of the system itself. Gas evolution and thermal lift would be expected to enhance the convective mass transport in the solution, but a through evaluation of these features of an operating system will require development of operational prototypes and comparison with a more complete model of such a system. Moreover, the choice of different levels of the tracking system (truly stationary, occasional tilt adjustment tracking or active diurnal tracking) is likely to be determined by the trade-off between the balance of systems cost and the efficiency of the solar concentrator, which is beyond the scope of this work.

The temperature profile of an actual operating system is highly dependent on the detailed construction of the cell, such as the particular encapsulation materials. Preliminary modeling results have suggested that a solar fuels generator system could operate at an elevated temperature $(\sim 330-350 \mathrm{~K})$ without using an active cooling system. ${ }^{13}$ However, the $10 \times$ concentrator designs exhibit a stronger temperature dependence of $\eta_{\text {STH }}$ than systems that do not use solar concentration. ${ }^{22}$ The trade-offs between the degradation of the PV performance and the enhancement of the solution transport and electrocatalysis as the temperature is increased depend strongly on the dimensions of the electrodes in the system. Specifically, for small electrode dimensions, at elevated temperatures the degradation of the performance of the PV materials dominates the entire cell performance (Figure 7). However, for large electrode dimensions, under $10 \times$ concentration, the reduction of the ohmic loss and catalytic overpotentials improves $\eta_{\mathrm{STH}}$ for the designs evaluated herein (Figure 7).

In both of the simulated designs, a $500 \mathrm{~nm}$ thick TCO layer having a sheet resistance of $20 \Omega / \square$ was applied on the top and the bottom of the photoabsorbers. This laterally conductive TCO layer facilitated a redistribution of current along the electrode width and reduced the ohimc loss in the system (Figure 3). In optimized cell designs, in which the electrode dimension is less than a few millimeters, $\eta_{\mathrm{STH}}$ was relatively insensitive to the presence or absence of the TCO layer. However, $\eta_{\mathrm{STH}}$ would be lowered by 1.0 percentage points for a large "trough" design $\left(l_{e}=1 \mathrm{~cm}, h_{e}=100 \mu \mathrm{m}, \xi=1 \%\right)$ operating at 350 $\mathrm{K}$ under detailed balance conditions.

From an equivalent circuit viewpoint, the efficiency of the integrated system would be identical to that of a stand-alone $\mathrm{PV}+$ electrolyzer system, if both systems utilized light absorbers, catalysts, and electrolyzers that exhibited mutually identical current vs voltage relationships. In the specific case considered herein, the low resistive loss and low operating current density of the spatially distributed, "internal" electrolyzer in the integrated cell designs yielded a higher electricity-to-fuel conversion efficiency than a traditional electrolyzer, which often operates $>1 \mathrm{~A} \mathrm{~cm}^{-2}$, due to the need to minimize the area-related balance of systems costs in stand-alone commercial electrolyzers. Therefore, the two optimized $10 \times$ concentrator designs, the "trough" design and the "bubble wrap" design, both outperformed the stand-alone PV+electrolyzer system on an efficiency basis when the same tandem photoabsorbers were utilized in each case. A full comparison between the two different system types would also clearly require an extensive cost analysis, but such is premature at this early 
stage of development of the type of integrated solar fuels generators evaluated herein.

A stand-alone PV+electrolyzer unit could in principle utilize a high-efficiency triple junction photovoltaic device, which can theoretically produce $>40 \%$ energy-conversion efficiency. The optimal band gaps, and operating voltages, of such triple junctions are significantly larger than the optimal values for use in an integrated solar-driven water splitting system, which is more well-suited to the use of tandem structures. An optimally performing triple-junction stand-alone PV+electrolyzer system would therefore clearly outperform the optically performing tandem-based integrated solar fuels generators considered herein. For comparison of both types of tandem-based systems, $\eta_{\mathrm{STH}}$ for the PV+electrolyzer is obviously dependent on the efficiency of the DC-to-DC converter as well as the efficiency of the stand-alone electrolyzer unit. For optimal performance, the stand-alone system would require a DC-DC converter that dynamically tracks the maximum power point of the PV array, and that also dynamically adjusts its output voltage and current to maintain optimal performance of the electrolyzer unit. Comparison of the data of Figures 4, 6 and 8, along with a linear increase of the efficiencies depicted in Figure 8 by higher assumed efficiencies for the electrolyzer and for the DC-DC converter in the stand-alone PV+electrolyzer combination, indicates that the optimized integrated system can in fact, with the physical electrode dimensions, membranes, electrolytes and system geometry described herein, provide comparable efficiency to that of an optimized, standalone PV+electrolyzer combination. The optimized $10 \times$ concentrator designs with smaller electrode dimensions described herein also permit the use of a semiconductor/liquid junction, in which no "buried" junction or lateral conductive TCO layer is required. The ability to utilize a stable semiconductor/liquid junction in the integrated design, e.g., a metal oxide/OER junction, would significantly broaden the materials choices relative to that available at present for a discrete PV unit, in which a solid-state "buried" junction is required.

\section{Conclusions}

In an integrated photoelectrolysis system that does not utilize solar concentration, maintaining low ohmic losses requires a maximum electrode width less than a few centimeters. In contrast, to maintain comparable ohmic losses, the integrated $10 \times$ concentrated devices modeled in this study require that the maximum electrode width or diameter must remain on the order of a few millimeters. The distribution of current density along the electrode width was observed to be nonuniform for the $10 \times$ concentrator designs, particularly for large cell dimensions. The overall $\eta_{\mathrm{STH}}$ of systems with smaller electrode dimensions showed a weaker dependence on the performance of the photoabsorbers and the catalysts than was the case for cells with larger dimensions. Minimal coverage of Nafion $(<1 \%$ of the cell area) was needed to maintain an ohmic loss of $<100 \mathrm{mV}$ in the bubble-wrap cell at the operating temperature of $350 \mathrm{~K}$, with an electrode diameter and height as large as $2 \mathrm{~mm}$ and $1 \mu \mathrm{m}$, respectively. With the same Nafion coverage and electrode height, when the electrode width in the trough design equaled the electrode diameter in the bubble-wrap design, the bubble-wrap design exhibited higher $\eta_{\mathrm{STH}}$ due to enhanced radial solution transport. At elevated operating temperatures, $\eta_{\mathrm{STH}}$ of cells with smaller dimensions decreased due to degradation of the performance of the photoabsorber materials, while $\eta_{\mathrm{STH}}$ of cells with larger dimensions increased with temperature due to enhanced transport in the solution and enhanced catalytic activity. The simulations also indicated that both optimized $10 \times$ concentrator designs (having small electrode dimensions) yielded comparable performance to an optimized PV + electrolyzer system, validating the merit of considering such integrated designs for implementation of a solar fuels generator that minimizes the materials utilization of the light absorbers and ionically conductive membranes in the system.

\section{Acknowledgments}

This material is based upon work performed by the Joint Center for Artificial Photosynthesis, a DOE Energy Innovation Hub, supported through the Office of Science of the U.S. Department of Energy under Award Number DE-SC0004993. The authors thank John C. Stevens for helpful discussion regarding the optics of solar concentrator designs. Authors Yikai Chen and Chengxiang Xiang contributed equally to this work.

\section{References}

1. Basic research needs for solar energy utilization, U.S. Department of Energy (DOE), Office of Basic Energy Sciences, Washington, D.C. (2005).

2. N. S. Lewis, Science, 315, 798 (2007).

3. M. G. Walter, E. L. Warren, J. R. McKone, S. W. Boettcher, Q. X. Mi, E. A. Santori, and N. S. Lewis, Chem Rev, 110, 6446 (2010).

4. A. J. Bard and M. A. Fox, Accts. Chem. Res., 28, 141 (1995).

5. B. A. Pinaud, J. D. Benck, L. C. Seitz, A. J. Forman, Z. B. Chen, T. G. Deutsch, B. D. James, K. N. Baum, G. N. Baum, S. Ardo, H. L. Wang, E. Miller, and T. F. Jaramillo, Energ Environ Sci, 6, 1983 (2013).

6. B. D. James, G. N. Baum, J. Perez, and K. N. Baum, Technoeconomic Analysis of Photoelectrochemical (PEC) Hydrogen Production, in, Directed Technologies (2009).

7. G. Peharz, F. Dimroth, and U. Wittstadt, Int. J. Hydrogen Energy, 32, 3248 (2007).

8. D. J. Friedman, R. R. King, R. M. Swanson, J. McJannet, and D. Gwinner, IEEE J Photovolt, 3, 1460 (2013).

9. D. C. Miller and S. R. Kurtz, Sol. Energ. Mat. Sol. C, 95, 2037 (2011).

10. A. Luque, J Appl Phys, 110, 031301 (2011).

11. R. Winston, J. C. Miñano, and P. G. Benitez, Nonimaging optics, Academic Press (2005).

12. J. J. O'Gallagher, Synthesis Lectures on Energy and the Environment: Technology, Science, and Society, 2, 1 (2008).

13. J. C. Stevens and A. Weber, Manuscript in preparation (2014).

14. J. Turner, Nat. Mater, 7, 770 (2008).

15. B. Parkinson, Sol. Cells, 6, 177 (1982)

16. S. Haussener, S. Hu, C. X. Xiang, A. Z. Weber, and N. S. Lewis, Energ. Environ. Sci., 6, 3605 (2013).

17. S. Haussener, C. X. Xiang, J. M. Spurgeon, S. Ardo, N. S. Lewis, and A. Z. Weber, Energ. Environ. Sci., 5, 9922 (2012).

18. S. Hu, C. X. Xiang, S. Haussener, A. D. Berger, and N. S. Lewis, Energ. Environ. Sci., 6, 2984 (2013).

19. J. M. Gregoire, C. X. Xiang, X. N. Liu, M. Marcin, and J. Jin, Rev. Sci. Instrum., 84 (2013).

20. S. M. Sze, Physics of Semiconductor Devices, John Wiley \& Sons, New York (1981).

21. A. J. Bard and L. R. Faulkner, Electrochemical Methods, Fundamentals and Applications, Wiley (2000).

22. S. Haussener, C. X. Xiang, J. M. Spurgeon, S. Ardo, N. S. Lewis, and A. Z. Weber, Energ. Environ. Sci., 5, 9922 (2012).

23. A. Rabl, Sol. Energy, 18, 93 (1975).

24. W. Shockley and H. J. Queisser, J. Appl. Phys., 32, 510 (1961).

25. C. H. Henry, J. Appl. Phys., 51, 4494 (1980).

26. H. E. Darling, Journal of Chemical \& Engineering Data, 9, 421 (1964)

27. H. Ito, T. Maeda, A. Nakano, and H. Takenaka, Int. J. Hydrogen Energy, 36, 10527 (2011).

28. Y. Sone, P. Ekdunge, and D. Simonsson, J. Electrochem. Soc., 143, 1254 (1996).

29. J. George and C. Menon, Surface and Coatings Technology, 132, 45 (2000).

30. K. Kinoshita, Electrochemical Oxygen Technology, New York: Wiley (1992).

31. N. Markovic, B. Grgur, and P. Ross, J. Phys. Chem. B, 101, 5405 (1997).

32. W. Sheng, H. A. Gasteiger, and Y. Shao-Horn, J. Electrochem. Soc., 157, B1529 (2010).

33. S. Siracusano, V. Baglio, A. Di Blasi, N. Briguglio, A. Stassi, R. Ornelas, E. Trifoni, V. Antonucci, and A. Arico, Int. J. Hydrogen Energy, 35, 5558 (2010).

34. A. T. Marshall, S. Sunde, M. Tsypkin, and R. Tunold, Int. J. Hydrogen Energy, 32, 2320 (2007)

35. F. Barbir, Sol. Energy, 78, 661 (2005).

36. J. Ivy, Summary of Electrolytic Hydrogen Production: Milestone Completion Report, p. Medium: ED; Size: 28 pp. pages (2004).

37. J. Newman, P. G. Hoertz, C. A. Bonino, and J. A. Trainham, J. Electrochem. Soc., 159, A1722 (2012). 